\title{
Novel Automated Production System for the Footwear Industry
}

\author{
Silvio Cocuzza ${ }^{1}$, Rosanna Fornasiero ${ }^{2}$, and Stefano Debei ${ }^{1}$ \\ ${ }^{1}$ CISAS "G. Colombo" - University of Padova, Padova, Italy \\ \{silvio.cocuzza, stefano.debei\}@unipd.it \\ ${ }^{2}$ ITIA-CNR, Milan, Italy \\ rosanna.fornasiero@itia.cnr.it
}

\begin{abstract}
The production process of most of the footwear companies working in the market segment of classic, casual, and fashion shoes is still handicraft. Moreover, the actual design and production processes are largely independent, and both of them need the physical prototypes of the semi-finished shoes in order to be accomplished. In this context, the IDEA-FOOT project introduces a new method for the shoe integrated design and production in which most of the production parameters are derived from the shoe 3D CAD model and an innovative automated production plant in which manipulators and automated machines are fully integrated. The aim of this paper is to present the main features of the automated plant, the advantages in using a new integrated design and production process, which is totally new for this market segment, and to analyze the impact of this new production model on the production performance and on the company organization.
\end{abstract}

Keywords: Footwear automation, production systems, robotics, CAD-CAM integration, production performance.

\section{Introduction}

Footwear companies working in the market segment of classic, casual, and fashion shoes, due to the global competition, need to reduce time to market and increase product diversification with small batches production, while keeping a high fashion and quality content of the product. The actual design and production processes are not suitable for this small batches and variegated production.

Indeed, the production process has still a handicraft connotation; most of the production operations are performed manually by workers, and even when automatic machines are used, the operators have to program them manually for each batch of shoes, and the semi-finished shoes and components handling is done by means of conventional conveyors that are manually loaded/unloaded.

Shoe manufacturing of classic, casual, and fashion shoes has a low degree of automation [1], mainly due to the complexity of the product and of the production process. On the other hand, the manufacturing of technical and sportwear shoes is highly automated. 
Several works can be found in the literature on the use of automation in different production stations of the shoe manufacturing, such as the automation of the lasting operation [2], of the grinding operation [3], of the roughing and cementing operations [2,4-6], of the bonding of the sole [7], of the finishing operations [2,8], and of the quality control [9]. In the EUROShoE project an automated production plant has been developed for the production of mass-customized shoes $[10,11]$.

The need of the physical prototypes of the semi-finished shoes to have the geometrical information necessary for the design and production phases is the main technological problem of the footwear companies of the considered market segment. Indeed, the actual design and production processes are largely independent, and both of them need the physical prototypes of the semi-finished shoes in order to be accomplished. In the design phase, the semi-finished shoe is necessary for the sequential development of the other shoe components to be assembled, whereas in the production process, the semi-finished product is necessary to program the semiautomatic machines for each batch of shoes.

In this context, the main objectives of the IDEA-FOOT project are:

- the introduction of a new method for the integrated design and production of the shoe, in which the key elements are the 3D CAD design of the shoe components and the transfer of the required geometrical information, tool paths, and production parameters from the design to the production process in a digital standard data format using a CAM S/W;

- the design, construction, test, and validation of the automated production plant in which the aforementioned method is implemented.

The proposed automated production plant is based on a production process that has been redesigned in order to reduce the complexity of the production and to increase flexibility, such as in the lean manufacturing approach [12].

In particular, the automated plant has been designed in order to have a flexible production both in terms of batch size (the minimum batch size is one shoe pair) and in terms of product style diversification.

This is in line with the agile and adaptive manufacturing approaches arisen at the beginning of the last decade as approaches to improve the competitiveness of the companies. Agile production is characterized by the integration of suppliers and customers in the value chain from the design to the production, to the marketing, and to the support services. There are many works applying different dimensions of the adaptive and agile paradigms (such as, for example in [13-16]), both from the point of view of technology improvement (implementation of flexible production systems, information and communication technology (ICT) tools, system integration) and people enhancement (competency management and training at all levels).

The aim of this paper is to present the main features of the automated plant, the advantages in using a new integrated design and production process, which is totally new for this market segment, and to analyze the impact of this new production model on the production performance and on the company organization. 


\section{New Automated Production System}

The automated production plant has been realized by means of integrating existing technologies and existing dedicated machines, which have been modified in order to work in an integrated way. All the production operations from the shoe lasting to the shoe bottom processing operations (up to the cementing of the shoe bottom) have been successfully automated.

In the automated plant, manipulators are used both for pick and place and for shoe bottom processing operations. A centralized control S/W, which has been customized for this project (DESMA), is used for the monitoring of the production and for the integration and synchronization of the dedicated production machines, the manipulators, the shoe last identification system (based on radio-frequency identification (RFID) technology), the oven, and the pallet conveyor.

The core of the automated plant is subdivided in two robotic cells: the robotic cell $\mathrm{A}$, in which research and development activities are involved, has been developed by CISAS, and the robotic cell B, in which state of the art technologies are involved, has been subcontracted to DESMA.

In particular, in the robotic cell A the two lasting machines (Brustia) receive the information on the article code for their automatic setup from an RFID chip inserted in the last, and moreover the heel seat and side lasting machine is loaded/unloaded by a manipulator (this was not possible for the toe lasting machine because a skilled operator is needed to assist that operation). On the other hand, in the robotic cell B some technologies already available in the market for the shoe bottom processing of technical and sportwear shoes have been modified for the scopes of this project.

The layout of the automated production plant, which have been defined comparing different solutions in terms of production time, work in progress (WIP), number of human operators, and required space, is presented in Fig. 1.

Before the robotic cell A, a human operator picks the last, the insole, and the upper from the related trolleys, assembles them together and puts the semi-assembled shoe in a trolley (station nr. 7 in Fig. 1).

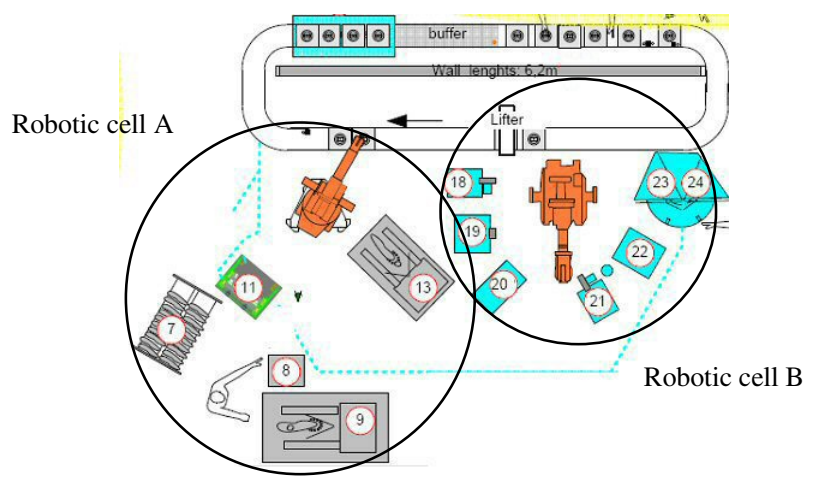

Fig. 1. Automated plant layout (Source: S. Cocuzza, Manipulation system for the toe lasting machine, EU FP7 IDEA-FOOT Project, Deliverable D 430.1, p. 5, 2012) 


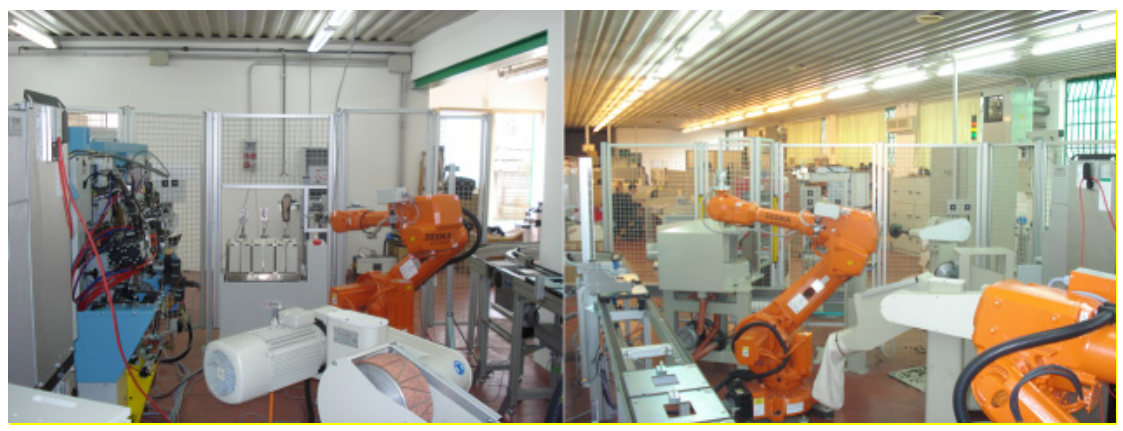

Fig. 2. Overall views of robotic cell A (left) and of robotic cell B (right)

(Source: S. Cocuzza, Manipulation system for the other machines of the integrated production cell, EU FP7 IDEA-FOOT Project, Deliverable D 440.1, p. 16-17, 2012)

In the robotic cell $\mathrm{A}$, first some manual operations are performed: a human operator picks the semi-assembled shoe from its trolley and puts it in a conditioning unit (nr. 8); after that operation, the operator places the last in front of an RFID station for the automatic setup of the toe lasting machine (nr. 9), performs the toe lasting operation, and then places the last in the man-robot exchange station (nr. 11), which is also a conditioning unit. Then, a manipulator is used to pick the last from the manrobot exchange station, place the chip of the last in front of a second RFID station for the automatic setup of the heel seat and side lasting machine (nr. 13), load and unload that machine, and finally place the last in the pallet conveyor.

In the robotic cell $\mathrm{B}$, a second manipulator is used to pick the last from the pallet conveyor (after that the last has been processed in the oven and that some optional manual operations have been performed, such as hot air application, cream application, and ironing), perform the shoe bottom processing operations (grinding, hammering, roughing, cleaning with compressed air, and cementing (nr. 18-22)), and then place it in the aspirator (nr. 23-24) to dry the water-based adhesive. The grinding, hammering, roughing, and cementing operations are performed by the robot, which presses the shoe bottom against the related ground-fixed rotating tool.

Two overall views of the robotic cell $\mathrm{A}$ and of the robotic cell $\mathrm{B}$ of the automated plant, which is installed at the BZ Moda premises, are presented in Fig. 2.

The main features of the new automated production plant are:

- use of manipulators to perform both pick and place and shoe bottom processing operations, thus reducing the processing time and the number of required human operators, and increasing the product quality;

- use of dedicated production machines (for the operations that cannot be performed by robots) that have been modified in order to reduce their setup time and to be loaded/unloaded by means of manipulators;

- implementation of a centralized control S/W for the monitoring of the production and the integration and synchronization of manipulators and automated machines.

The above presented approach represents a solution that can be rapidly adopted by the footwear companies of the considered market segment, in a smooth and gradual change toward automation. 
Existing dedicated production machines have been modified in their mechanical and communication interfaces in order to be integrated with the rest of the automated plant and served by manipulators, to receive the setup parameters and tool paths generated automatically by the CAM S/W, and for their automatic setup during the production by using RFID systems.

An RFID tag is used to store in each last the batch information that is called up, when required, to guide the manipulators or the automated machines. The identification code of each shoe model is used in order to recall the production parameters and tool paths from the local databases of the machines and manipulators for their automatic setup in function of the shoe model.

The daily productivity of the automated pilot plant is approximately 320 [pairs/shift] ( 1 shift $=8$ hours), and only 5 human operators are required (considering all the production operations from assembling the last with the insole and the upper up to delasting, excluding the aforementioned optional manual operations).

In the traditional plant with classic conveyor line taken as a reference, 13 human operators are needed, and the daily productivity is 554 [pairs/shift]. In the proposed automated plant, if 2 manipulators are used in the shoe bottom processing robotic cell, the daily productivity can be increased up to 600 [pairs/shift] and the number of human operators can be reduced to 7 . Productivity can be additionally increased by adding more manipulators and/or duplicating production stations at the bottlenecks.

Once that the automated plant has been realized, it has been interfaced with the rest of the traditional production plant (including sole application, heel application, finishing, and delasting stations) and small batches of shoes have been produced for validation purposes. The analysis of the produced shoes evidenced that increased quality production operations have been performed, which turned out in high quality finished shoes.

\section{Impact on Production Performance and on Company Organization}

The implementation of the proposed integrated design and production model will contribute to enhance the competitive strength of the footwear companies through leveraging and optimizing the value of knowledge within and across companies by supporting the creation of a sustainable industrial production process. In particular, the solution proposed in this project goes both in the direction of economic sustainability, allowing companies to reduce production costs, and towards environmental and social sustainability, going beyond the lean paradigm.

Time to market will be significantly reduced and footwear companies will have the possibility to be more flexible and responsive in the development of new shoe models. The new production system is extremely flexible both in terms of production of small batches (up to one shoe pair) and in terms of product diversification, with very limited setup time to manage product variants.

Moreover, the relationship between the shoe producer and its suppliers is totally revised: the design of new shoe models will be mainly carried out by the shoe producer with limited intervention of subcontractors, and the production of the shoe 
components will be made concurrently by the suppliers, saving up to $50 \%$ of the design and development time with respect to the actual sequential development of the shoe components based on physical prototypes.

The impact will be on three major dimensions of sustainability: economic, social, and environmental.

Concerning the economic sustainability, an important reduction of the production costs and time to market is envisioned. Moreover, the implementation of these new technologies allows to maintain the production (or, at least, part of it) in the origin country reducing delocalization and assuring workplaces.

Concerning the social sustainability, the introduction of the new integrated design and production method requires the development of new skills and competences both in the area of design, where people have to learn to use new CAD modules, and in the area of production, where workers are asked to do added-value operations in the automated plant. Moreover, the introduction of the new automated production plant improves working conditions: operations that require physical efforts and repetitive manipulations are performed by manipulators, workers are no more involved in risky operations, and the direct contact with harmful substances is eliminated (toxic adhesives have been substituted by water-based adhesives and the operations where leather powder can be breathed causing health problems are carried out in a protected area).

The environmental sustainability of the production processes is nowadays a relevant issue requiring that companies manage the negative externalities caused by their production. In the framework of this project, thanks to the innovative production system, scraps and material waste during the production phase can be significantly reduced (the integration of the design and production phases allows a better organization of the shoe development) and toxic adhesives and solvents are eliminated (water-based adhesives are used).

Based on qualitative information gathered from company managers and technology providers, the analysis of the impact of the implemented technologies on the production performance has been carried out, which can be summarized as follows:

- Impact on flexibility. The production is highly flexible both in terms of batch size (the minimum batch size is one shoe pair) and in terms of product style diversification.

- Impact on productivity. The automated plant productivity is about 320 [pairs/shift]. Nevertheless, productivity can be increased by adding more manipulators and/or duplicating production stations at the bottlenecks.

- Impact on quality. Defects are reduced thanks to the increased accuracy of the automated operations. The product quality is also improved thanks to the upstream improvement in the more precise product design.

- Impact on balancing of production flow. Bottlenecks are eliminated and WIP is minimized thanks to an accurate design of the automated plant and of the centralized control S/W.

- Impact on waiting periods. Waiting periods due to inadequate manufacturing schedules and long production stops for the machines setup are eliminated. The production system is based on an initial plant setup for all the planned production models, which allows to have a seamless flow of production. 
- Impact on motion waste. The automated plant design has been optimized so that any operation of workers or machines identified as non-value-adding action is eliminated.

- Impact on capability to find errors. The centralized control S/W generates an alert any time there is a problem in the production so that the worker knows where the problem is located and what caused it.

\section{Conclusions}

The IDEA-FOOT project introduces a new method for the integrated design and production of shoes and an innovative automated production plant in which manipulators and automated machines are fully integrated. Most of the production parameters and tool paths are derived from the shoe 3D CAD model and then transferred to the production using a dedicated CAM S/W. The production data is then recalled in the manipulators and in the production machines by means of RFID systems, thus reducing their setup time. In the automated plant, all the production operations from the shoe lasting to the shoe bottom processing operations (up to the cementing of the shoe bottom) have been successfully automated. A centralized control S/W is used for the monitoring of the production and for the integration and synchronization of manipulators and automated machines. The plant productivity is about 320 [pairs/shift] ( 1 shift $=8$ hours), which can be increased up to 600 [pairs/shift] by adding one manipulator. Productivity can be additionally increased by adding more manipulators and/or duplicating production stations at the bottlenecks. Once that the automated plant has been realized, it has been interfaced with the rest of the traditional production plant and it has been validated by producing small batches of high quality shoes, which demonstrated the accuracy of the production operations. The presented approach, which is totally new for this market segment, allows the reduction of production costs and time to market, with a highly flexible production both in terms of batch size (the minimum batch size is one shoe pair) and in terms of product style diversification. The introduction of automation implies a reduction of the number of human operators required, thus reducing production costs. On the other hand, more qualified workers are needed and their working conditions are improved. Thanks to the new integrated design and production process based on the shoe 3D CAD model, the relationship between the shoe producer and its suppliers is totally revised: the design of new shoe models will be mainly carried out by the shoe producer, and the production of the shoe components will be made concurrently by the suppliers, saving up to $50 \%$ of the design and development time. Finally, the improved quality of the production operations implies a significant reduction of scraps and material waste during the production.

Acknowledgement. The results presented in this paper have been obtained in the framework of the IDEA-FOOT project (Innovative DEsign and mAnufacturing systems for small series production for European FOOTwear companies) - 7th Framework Programme - Research for the benefit of SMEs - FP7-SME-2008-I Project n. 232585. 


\section{References}

1. Ronthaler, M., de Gea Fernandez, J., Vogele, T.: Automation in the Footwear Industry Innovative Robotics to Support Complex Artisan Production. Industrie Management 1, 59-61 (2011)

2. Nemec, B., Žlajpah, L.: Automation in shoe assembly. In: Proceedings of the 15th International Workshop on Robotics in Alpe-Adria-Danube Region (RAAD 2006), Balatonfüred, Hungary, pp. 131-136 (2006)

3. Nemec, B., Zlajpah, L.: Shoe grinding cell using virtual mechanism approach. In: Inst. for Syst. and Technol. of Inf. Control and Commun. (ed.), Proceedings of the 5th International Conference on Informatics in Control, Automation and Robotics (ICINCO 2008), Setubal, Portugal, pp. 159-164 (2008)

4. Hu, Z., Marshall, C., Bicker, R., Taylor, P.: Automatic surface roughing with 3D machine vision and cooperative robot control. Robotics and Autonomous Systems 55(7), 552-560 (2007)

5. Rooks, B.W.: Robots bring automation to shoe production. Assembly Automation 16(3), 22-25 (1996)

6. Kochan, A.: Actis and the shoe industry. Assembly Automation 16(3), 30-31 (1996)

7. Tae-Jung, L., Pil-Gyu, P., Jong-Chul, S., Dong-Joo, P., Hee-Tae, A.: A study on development of 3D outsole profile scanner for footwear bonding automation. In: Inst. Control, Autom. \& Syst. Eng., Proceedings of the 2001 International Conference on Control, Automation and Systems (ICCAS 2002), Taejon, South Korea, pp. 2857-2860 (2001)

8. Choi, H.-S., Hwang, G.-D., You, S.-S.: Development of a new buffing robot manipulator for shoes. Robotica 26(1), 55-62 (2008)

9. Alcantud, J.A.L., Carbonell, A.A., Asensi, G.D., Balibrea, L.-M.T.: Inspection and quality control based on artificial vision techniques for the automation of manufacturing process in the footwear industry, Proceedings of the Second Asian Conference on Computer Vision (ACCV '95), Ed. In: Li, S., Teoh, E.-K., Mital, D., Wang, H. (eds.) ACCV 1995. LNCS, vol. 1035, pp. 685-689. Springer, Heidelberg (1996)

10. Dulio, S., Boër, C.: Integrated production plant (IPP): an innovative laboratory for research projects in the footwear field. International Journal of Computer Integrated Manufacturing 17(7), 601-611 (2004)

11. Boër, C., Dulio, S.: Mass Customization and Footwear - Myth, Salvation or Reality?. Springer (2007)

12. Womack, J.P., Jones, D.T., Roos, D.: The Machine That Changed the World: The Story of Lean Production. HarperBusiness (2003)

13. Ashall, D., Parkinson, B.: Leaning towards Agile. Manufacturing Engineer 81(1), 27-32 (2002)

14. Gunasekaran, A.: Agile Manufacturing: A Framework for Research and Development. International Journal of Production Economics 62(1), 87-105 (1999)

15. Sharifi, H., Colquhoun, G., Barclay, I., Dann, Z., Agile Manufacturing: A Management and Operational Framework, Proceedings of the Institution of Mechanical Engineers, vol. 215-B, pp. 857-869, 2001.

16. Dashchenko, A.: Manufacturing Technologies for Machines of the Future - 21st Century Technologies. Springer (2003) 